\title{
Evaluation and outcomes of paediatric epilepsy surgery in Singapore: A single-centre audit
}

\author{
${ }^{1}$ Si Qi Tan $B S c$ MD, ${ }^{2}$ Adeline Seow Fen Ngoh mBBS мRCPCH, ${ }^{3}$ Wai Hoe Ng MD FRACS, \\ ${ }^{4}$ Wanying Xie $F R C R$ MMed, ${ }^{2}$ Janardhan Krishnappa $M B B S$ MRCPCH, ${ }^{1}$ Nazima Binte Sahul \\ Hamed $B S c,{ }^{5}$ Phua Hwee Tang FRCR MMed, ${ }^{6}$ Tchoyoson Choie Cheio Lim FRCR MMed, \\ ${ }^{7}$ Kenneth Tou En Chang $M B C h B, F R C P a t h,{ }^{8}$ Ming Lee $M_{B C h B},{ }^{9}$ Martha I-Ming Kao BHSc (Nurs) \\ $\operatorname{Grad} \operatorname{Dip}($ Nurs $),{ }^{3,10}$ David Chyi Yeu Low MRCS FRCSEd(SN), ${ }^{2}$ Derrick Wei Shih Chan $B M B S$ MRCPCH
}

${ }^{1}$ Department of Paediatrics, KK Women's and Children's Hospital; ${ }^{2}$ Department of Neurology, KK Women's and Children's Hospital; ${ }^{3}$ Department of Neurosurgery, National Neuroscience Institute; ${ }^{4}$ Department of Nuclear Medicine and Molecular Imaging, Singapore General Hospital; ${ }^{5}$ Department of Diagnostic and Interventional Imaging, KK Women's and Children's Hospital; 'Department of Neuroradiology, National Neuroscience Institute ${ }^{7}$ Department of Pathology and Laboratory Medicine, KK Women's and Children's Hospital; ${ }^{8}$ Department of Pathology, Singapore General Hospital; ${ }^{9}$ Nursing Clinical Services - Neurology, KK Women's and Children's Hospital; ${ }^{10}$ Department of Neurosurgery, KK Women's and Children's Hospital, Singapore

\begin{abstract}
Background: Paediatric epilepsy surgery reduces seizure burden in drug-refractory epilepsy reducing long-term neurocognitive damage. Methods: Single-centre retrospective audit of pre-surgical evaluations and outcomes of the paediatric epilepsy and epilepsy surgery programme over eleven years at KK Women's and Children's Hospital, Singapore. Data were collected based on National Institute of Neurological Disorders and Stroke Common Data Elements guidelines. Outcome was categorized using Engel classification scale, and favourable outcome defined as greater than $50 \%$ decrease in seizure frequency or drop attacks. Results: Thirty-three children underwent epilepsy surgery, with mean follow-up 3.8 \pm 3.1 years. Median age at surgery was 10.9 years. Twenty-four children with focal epilepsy underwent resection of the epileptogenic focus, including lesionectomy $(n=8)$, anterior temporal lobectomy $(n=7)$, extratemporal lobectomy $(n=7)$ and hemispherectomy $(n=2)$. Nine children underwent corpus callosotomy for Lennox Gastaut Syndrome $(n=8)$ and West Syndrome $(n=1)$. Median hospital stay duration was ten days. All twenty-three focal epilepsy patients with minimum three-month follow-up achieved greater than 50\% seizure reduction. Fifteen (65\%) focal epilepsy patients achieved seizure-freedom (Engel Class IA) after first surgeries. Four patients required second surgeries, with two achieving seizure-freedom. Intraoperative MRI (iMRI) is beneficial. All nine corpus callosotomy patients $(100 \%)$ achieved greater than $50 \%$ decrease in drop attacks. Number of antiepileptic drugs was weaned for 21/32 (66\%) patients. Post-operative complications were low and some patients had anticipated neurological deficits. Outcomes were comparable to current literature.

Conclusions: In well-selected candidates with tailored evaluation, paediatric epilepsy surgery is a safe therapeutic option with favourable outcomes and can be performed across the entire paediatric age range.
\end{abstract}

Keywords: Drug-refractory epilepsy, Epilepsy surgery, Focal epilepsy, Paediatric epilepsy

\section{INTRODUCTION}

Poorly-controlled epilepsy in children is detrimental to the developing brain. ${ }^{1}$ One third of children with epilepsy have drug-refractory epilepsy (DRE) ${ }^{2,3}$, defined as poor seizure control despite trials of two tolerated, appropriately chosen and used antiepileptic drugs (AED) ${ }^{4}$ In paediatric focal epilepsy, DRE is particularly common, especially if an epileptogenic lesion is identified on neuroimaging ${ }^{5,6}$ and only $32.7 \%$ with abnormal neuroimaging achieve spontaneous remission, defined as five years seizure- and

Address correspondence to: Tan Si Qi, KK Women's and Children's Hospital, 100 Bukit Timah Rd, Singapore 229899. Tel (65) 9126 4282, Email: siqi. $\tan @$ mohh.com.sg

Date of Submission: 4 February 2021; Date of Acceptance: 8 June 2021

https://doi.org/10.54029/2021zpd 
medication-free ${ }^{10}$. Epilepsy surgery is currently the only cure in focal DRE..$^{7-9}$ Randomized controlled trials of epilepsy surgery in adult temporal lobe epilepsy and paediatric focal epilepsy showed 58\% seizure-freedom versus $8 \%$ treated medically ${ }^{11}$ and $77 \%$ seizure-freedom versus $6 \%$ treated medically ${ }^{12}$ respectively. Early epilepsy surgery improves rehabilitation potential, prevents further developmental damage and is more cost-effective long term ${ }^{8,10,12}$ but is under-utilized. ${ }^{13}$

Epilepsy surgeries can be curative or palliative. Curative surgery includes lesionectomy, temporal or extratemporal lobectomy, hemispherotomy, in which the epileptogenic zone is neurologically disconnected but left intact, or hemispherectomy. Palliative surgery aims to reduce seizure burden and negative sequelae and includes corpus callosotomy, to disrupt interhemispheric seizure spread thereby decreasing drop attacks that otherwise cause falls and trauma ${ }^{14}$, and Vagus Nerve Stimulator (VNS) implantation.

We audited paediatric epilepsy surgeries performed KK Women's and Children's Hospital $(\mathrm{KKH})$ in Singapore, pre-surgical evaluation and surgical outcomes.

\section{METHODS}

\section{Study settings and patient selection}

We audited all drug-refractory epilepsy (DRE) patients who underwent epilepsy surgery at KK Women's and Children's Hospital in Singapore between January 2009 and October 2020.

Demographics, age of seizure onset, school status, age at time of surgery, and relevant medical histories were collected from patient records. Seizure semiology was recorded from clinical history and electroencephalogram (EEG) reports and categorized according to the 2017 ILAE seizure and epilepsy syndrome classification. ${ }^{15}$ Participant and disease characteristics, neuroimaging, EEG, surgery and pathology data were classified in accordance with the National Institute of Neurological Disorders and Stroke Common Data Elements guidelines for epidemiologic studies of epilepsy. ${ }^{16}$ Confidentiality was maintained according to institutional regulations. Ethics approval was not required as this study was an institutional audit.

\section{Pre-surgical evaluation and selection for surgery}

All patients had undergone investigation with continuous video EEG and magnetic resonance imaging (MRI). Interictal and ictal epileptiform discharges were classified concordant if $\geq 75 \%$ of discharges corresponded to predicted seizure focus. In patients with multiple seizure types the most common seizure was analysed.

Selected patients with unclear localization on EEG or lesion-negative MRI scans underwent 18F-Fluorodeoxyglucose (FDG) position emission tomography (PET) scans to localize epileptogenic zones. In patients whose resection plan potentially included eloquent cortex, functional magnetic resonance imaging (fMRI) was performed to evaluate motor and language function to predict post-operative deficits. Patients of school age also underwent neuropsychological evaluation. Suitable candidates for resective or palliative surgery were counselled by the paediatric epileptologist and paediatric neurosurgeon, with pre-surgical planning performed jointly.

\section{Surgical approach and histological classification}

Operative findings and extent of resection were obtained from records and post-operative imaging. Surgical resection included standard temporal lobectomy, tailored lobectomies, lesionectomies, anatomical hemispherectomy, and corpus callosotomies. Where possible, intra-operative MRI (iMRI) was used to guide resection. Intraoperative electrocorticography $(\mathrm{ECoG})$ was also utilized in selected cases to further delineate the epileptogenic zone. In all cases the epileptologist and epilepsy neurosurgeon confirmed the resection plan pre-operatively, evaluated the extent of resection intra-operatively (with use of ECoG and iMRI scans where indicated or available) and determined the resection margins together, balancing resection extent to optimize seizure control and minimize deficits.

Histological classification was extracted from clinical histopathology reports. FCD was categorized according to the recent ILAE classification scheme. ${ }^{17}$ Other pathological findings and tumour subtypes were described based on World Health Organization classification. ${ }^{18}$

\section{Surgical outcomes}

Post-operative complications were classified into (1) minor reversible medical complications such as metabolic disturbances, post-operative fever, cerebrospinal fluid leak, infections, aseptic meningitis, and intracranial hematomas, (2) minor neurological complications such as post-operative seizures or transient neurological deficits resolving within three months, and (3) major medical and neurological complications such as hydrocephalus 
or abscesses requiring intervention, or unexpected neurological deficits persisting beyond three months. Length of hospital stay and need for re-operation were also recorded.

Outcome was recorded based on the last paediatric neurologist consult and categorized using the Engel classification system. ${ }^{19}$ Patients with minimum three months follow-up were included in outcome analysis. Favourable outcome was defined as greater than 50\% decrease in frequency of seizures or drop attacks. Postoperative AEDs, date of seizure recurrence, and qualitative descriptions were also recorded.

\section{Statistical analyses}

Descriptive statistical analyses were performed using Microsoft Excel.

\section{RESULTS}

Patient demographics and baseline characteristics

Thirty-three patients (19 males, 14 females) with DRE underwent epilepsy surgery - Chinese $(n=20,61 \%)$, Malay $(n=8,24 \%)$, Indian $(n=1$, $3 \%)$ and others $(n=4,12 \%)$. Thirty-two $(97 \%)$ had minimum of three months post-surgical outpatient follow-up (mean $3.9 \pm 3.1$ years). Mean age was $3.4 \pm 4.6$ years (range 8 hours of life-16 years) at seizure onset and $10.2 \pm 6.5$ years (range 3 months-25 years) at surgical evaluation. Mean duration of epilepsy prior to operation was 7.2 \pm 6.4 years (range 11 days-24 years). Mean age of surgery was $10.6 \pm 6.7$ years (range 5 months- 25 years, median 10.9 years).

Most (24/33 (73\%)) had focal epilepsy: eight temporal lobe (24\%), six frontal lobe (18\%), four occipital lobe (12\%), two parietooccipital $(6 \%)$, one parietal lobe $(3 \%)$ and four hemispheric (12\%). Eight (24\%) had LennoxGastaut syndrome (LGS) and two (6\%) had West Syndrome (WS). Five (15\%) had associated underlying diagnoses: three Tuberous Sclerosis (TS), one Sturge-Weber Syndrome (SWS) and one FCD with Sodium Voltage-Gated Channel Alpha Subunit 1 (SCN1A) mutation.

Clinical phenotype and epilepsy diagnoses are listed in Table 1 and Supplementary Table 1. Seizure frequency ranged from once a year (in a patient with high probability of cure with epilepsy surgery) to refractory status epilepticus. Sixteen (48\%) had global developmental delay, and twelve (36\%) were cognitively or language delayed. Seventeen (51\%) patients had abnormal neurological examination pre-operatively, and six (18\%) exhibited behavioural difficulties with autistic features. Twelve (36\%) attended normal school, $12(36 \%)$ attended special school, one (3\%) was home-schooled, one (3\%) attended preschool and seven $(21 \%)$ were infants and pre-schoolers.

Patients trialled a mean of five AEDs prior to surgery. Four had used the ketogenic diet but did not tolerate it or showed no benefit.

\section{Pre-surgical evaluations}

Table 1 and Supplementary Table 2 included presurgical radiological and EEG evaluations. Ten patients (30\%) had 18F-FDGPET scans performed, with 8 showing interictal focal hypometabolism and 2 showing focal hypermetabolism over the epileptogenic foci. The latter two had electrographic seizures on pre-PET EEG and were classified as ictal. Seven patients $(21 \%)$ had language fMRI. An example of pre-surgical evaluation is illustrated in Figure 1.

The most common aetiology was structural $(76 \%) ; 14(42 \%)$ focal cortical dysplasia (FCD), two (6\%) mesial temporal sclerosis, five $(15 \%)$ ganglioglioma, oligodendroglioma or dysembryoplastic neuroepithelial tumour (DNET), three (12\%) TS tubers (12\%), one (4\%) SWS (4\%), and one with inflammation (4\%) (Figure 2). In the focal epilepsy group, 22/24 (92\%) had congruent lesions on MRI.

Eight patients had LGS and two had WS, with all undergoing corpus callosotomy except for one WS patient who had hemimegalencephaly and underwent hemispherectomy. Seventeen patients underwent VNS implantation and are not described further here.

In focal epilepsy patients, EEG interictal discharges lateralized to the seizure focus in 21/24 (88\%) (Table 1). Ictal EEG in all 24 were localizing, with five (20\%) having secondary generalization. EEG in patients with WS and LGS were consistent with the clinical syndrome, with electroclinical seizures captured in 6/8 (75\%).

\section{Epilepsy surgeries performed}

Epilepsy surgery $(n=33)$ comprised eight lesionectomies (24\%), seven anterior temporal lobectomies with amygdalohippocampectomy $(21 \%)$, four unilobar extratemporal lobectomies $(16 \%)$, three multilobar extratemporal lobectomies (9\%), two hemispherectomies (6\%) and nine corpus callosotomies $(27 \%)$. Of these surgeries $(n=33)$, nine $(27 \%)$ utilized iMRI and five $(15 \%)$ utilized ECoG. 


\begin{tabular}{|c|c|c|c|c|c|c|c|c|c|c|c|c|c|c|}
\hline 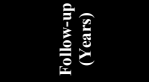 & $f$ & $n$ & 8 & $n$ & 古 & $\stackrel{\circ}{\circ}$ & $\dot{\infty}$ & r & $\bar{n}$ & a & \pm & $=$ & 3 & - \\
\hline 焉鄫 & $\unlhd$ & $\unlhd$ & $\unlhd$ & $\leqq$ & $\leqq$ & $\leqq$ & $\leq$ & $\leq$ & $\leq$ & $\leqq$ & $\unlhd$ & $\cong$ & $\unlhd$ & $\leq$ \\
\hline 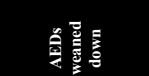 & $\stackrel{\circ}{=}$ & $\stackrel{3}{:}$ & $\mathscr{y}$ & $\stackrel{0}{=}$ & $\stackrel{\circ}{2}$ & $\stackrel{\circ}{=}$ & $\stackrel{\mathscr{s}}{=}$ & $\stackrel{\infty}{=}$ & $\stackrel{\circ}{:}$ & $\stackrel{2}{z}$ & $\stackrel{8}{=}$ & \& & $\stackrel{\circ}{z}$ & $\stackrel{8}{:}$ \\
\hline 今心 & $\stackrel{8}{=}$ & $\stackrel{20}{2}$ & 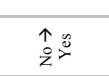 & 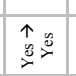 & 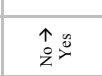 & $\stackrel{8}{=}$ & $\begin{array}{l}\hat{1}: g \\
\ddot{2}: 0\end{array}$ & $\stackrel{5}{x}$ & 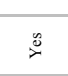 & $\ddot{:}$ & $\ddot{n}$ & 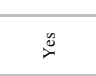 & $\stackrel{8}{x}$ & 3 \\
\hline 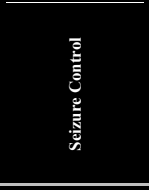 & 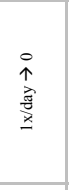 & 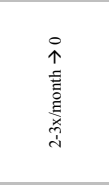 & 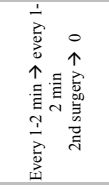 & 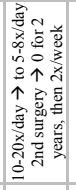 & 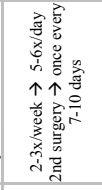 & 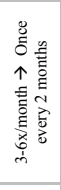 & 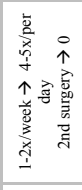 & $\begin{array}{l}\text { i } \\
\text { 麗 } \\
\underline{\underline{z}}\end{array}$ & 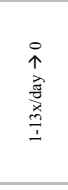 & 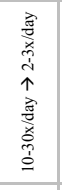 & 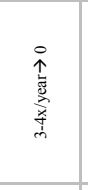 & 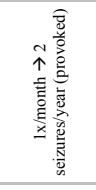 & 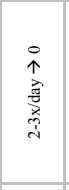 & 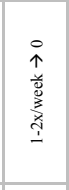 \\
\hline 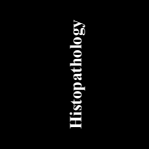 & 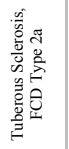 & 总 & 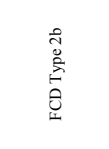 & 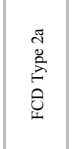 & 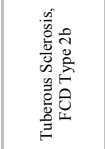 & 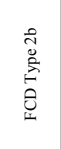 & 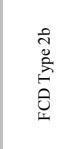 & 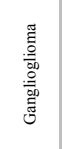 & 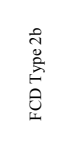 & 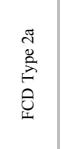 & 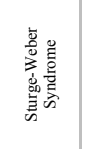 & 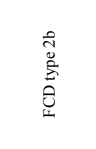 & $\frac{\frac{n}{0}}{\frac{0}{5}}$ & 产 \\
\hline 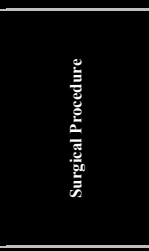 & 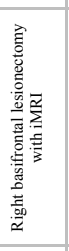 & 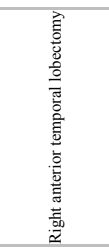 & 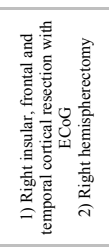 & 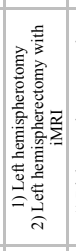 & 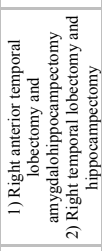 & 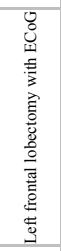 & 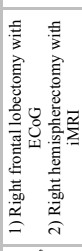 & 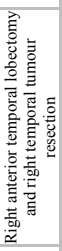 & 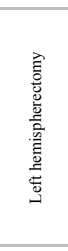 & 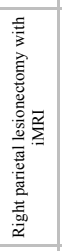 & 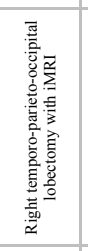 & 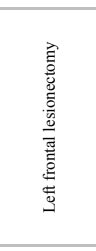 & 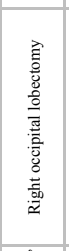 & 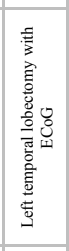 \\
\hline$\frac{d}{3}$ & $\approx$ & $\stackrel{5}{5}$ & $\stackrel{y}{=}$ & $\stackrel{s}{x}$ & $\stackrel{8}{x}$ & $\stackrel{8}{5}$ & 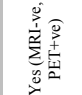 & $\stackrel{8}{=}$ & $:$ & 3 & 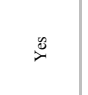 & $\mathscr{x}$ & 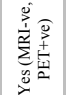 & $\approx$ \\
\hline 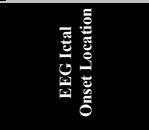 & 志 & $\infty$ & $\infty$ & 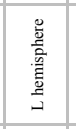 & $\frac{d}{d}$ & 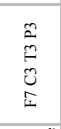 & 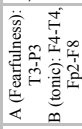 & 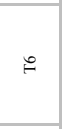 & 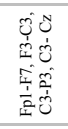 & \pm & 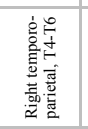 & $\begin{array}{l}3 \\
\text { s } \\
1\end{array}$ & $\stackrel{\circ}{\circ}$ & $\stackrel{n}{\mu}$ \\
\hline 尊耪 & 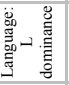 & 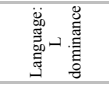 & . & & . & 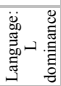 & & . & . & . & & 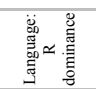 & . & . \\
\hline 高 & 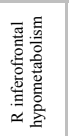 & 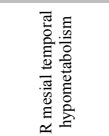 & 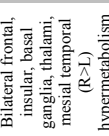 & 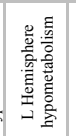 & 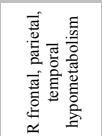 & 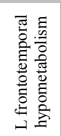 & 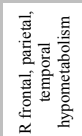 & & & . & & & & \\
\hline 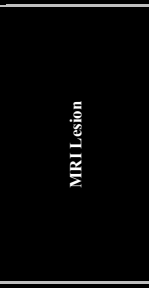 & 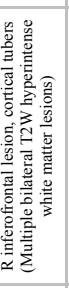 & 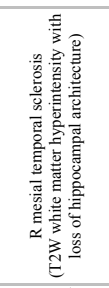 & 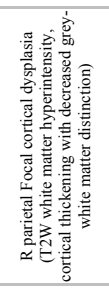 & 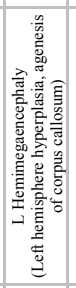 & 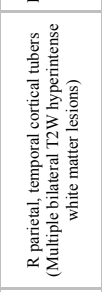 & 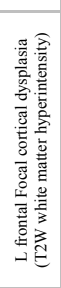 & 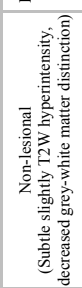 & 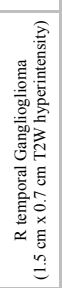 & 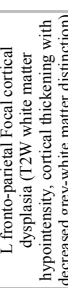 & 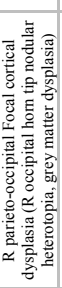 & 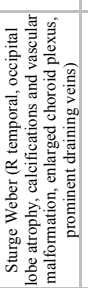 & 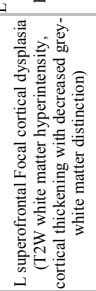 & 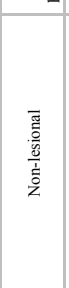 & 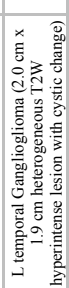 \\
\hline 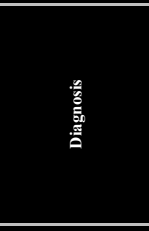 & 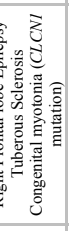 & 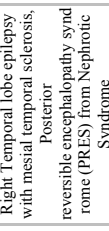 & 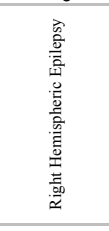 & 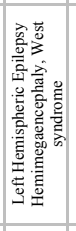 & 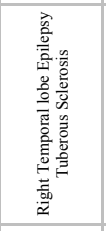 & 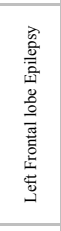 & 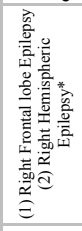 & 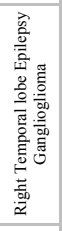 & 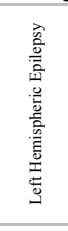 & 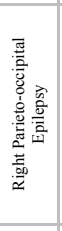 & 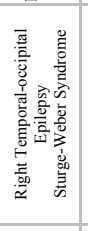 & 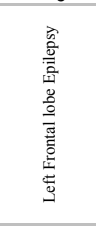 & 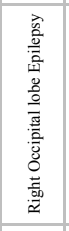 & 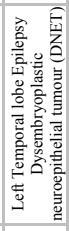 \\
\hline 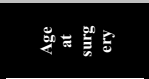 & 吾 & 㞗 & 㞗 & $\geq$ & 鹿 & 表 & sे & 言 & $\Xi$ & 量 & 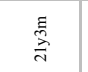 & 鹿 & 昱 & 2 \\
\hline 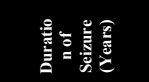 & $\approx$ & $\cong$ & 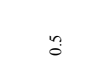 & 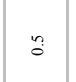 & 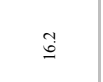 & 8 & $\infty$ & के & $n$ & $=$ & $\stackrel{9}{\pi}$ & 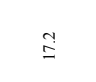 & $\stackrel{\infty}{\stackrel{\infty}{\infty}}$ & in \\
\hline 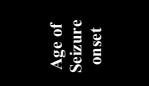 & 高 & $\bar{D}$ & 言 & छ & $\Xi$ & $\frac{\bar{g}}{2}$ & 高 & $\geq$ & 啇 & $\geq$ & घ & 品 & $\Xi$ & $\stackrel{\Xi}{\Xi}$ \\
\hline$n \cdot x$ & ш & $\Sigma$ & 山 & $\Sigma$ & $\Sigma$ & " & 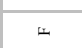 & 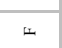 & i & $\Sigma$ & 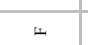 & $\Sigma$ & 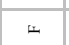 & $\bar{z}$ \\
\hline 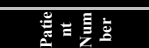 & - & $\sim$ & m & + & in & $\circ$ & r & $\infty$ & $a$ & $\cong$ & $=$ & $\simeq$ & $=$ & \pm \\
\hline
\end{tabular}




\begin{tabular}{|c|c|c|c|c|c|c|c|c|c|c|c|c|c|c|c|c|c|c|c|c|c|c|c|}
\hline$\overbrace{0}^{\infty}$ & $\stackrel{\infty}{\infty}$ & $\stackrel{n}{3}$ & & 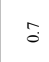 & $\stackrel{\infty}{0}$ & 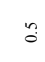 & & & â & 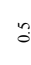 & 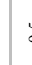 & & & 3 & 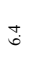 & $\underset{\mathrm{d}}{\stackrel{\circ}{ }}$ & สี & $y$ & & & r & & 㞼 \\
\hline$\leq$ & $\leq$ & $\leq$ & & $\leq$ & $\leqq$ & $\unlhd$ & & & $\leq$ & $\leq$ & & & E & $\leqq$ & $\leq$ & 吕 & $\leqq$ & 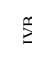 & & & $\leqq$ & & 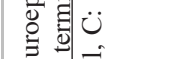 \\
\hline$\stackrel{g}{\check{2}}$ & $\stackrel{2}{2}$ & $\stackrel{8}{=}$ & & $\stackrel{2}{z}$ & $\stackrel{2}{z}$ & $y^{3}$ & & & $\check{z}$ & $\ddot{s}$ & & & 2 & 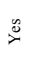 & $\check{x}_{x}^{2}$ & $\stackrel{2}{z}$ & $\stackrel{s}{x}$ & & & & $\stackrel{\gtrless}{z}$ & 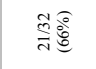 & 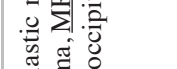 \\
\hline$\stackrel{8}{\check{2}}$ & $\ddot{x}$ & 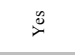 & & $\stackrel{0}{:}$ & 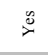 & $\stackrel{8}{0}$ & & & $=$ & $y$ & & & 8 & $\stackrel{8}{=}$ & $\stackrel{g}{\nearrow}$ & $\stackrel{8}{\circ}$ & 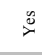 & 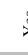 & & & $\stackrel{g}{\check{r}}$ & 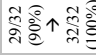 & \\
\hline 勇 & 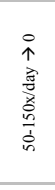 & 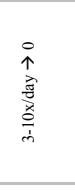 & 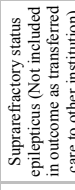 & 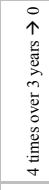 & 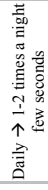 & $\begin{array}{l}0 \\
\uparrow \\
\text { 离 } \\
\text { 章 } \\
\text { 常 }\end{array}$ & & & 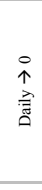 & & 要 & & & 政 & & & $\begin{array}{l}0 \\
0\end{array}$ & & & & 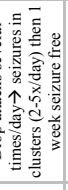 & & 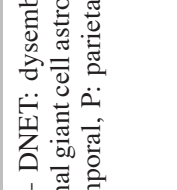 \\
\hline 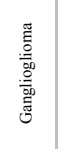 & 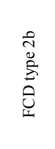 & 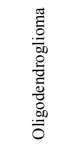 & 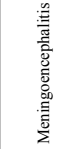 & 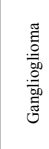 & 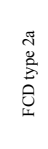 & 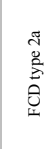 & & & 镐 & $\frac{0}{2}$ & & & & . & & & & & & & 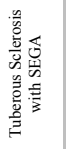 & & 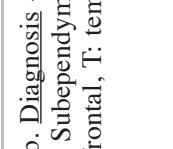 \\
\hline 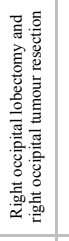 & 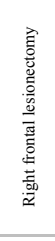 & 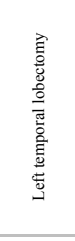 & 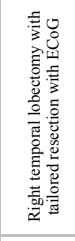 & 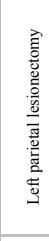 & 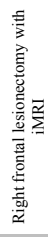 & 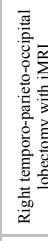 & & & 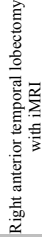 & : & & & is & 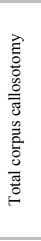 & 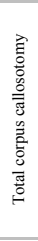 & 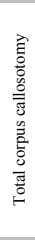 & 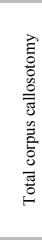 & (1) & 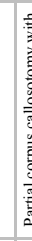 & & 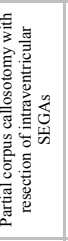 & & 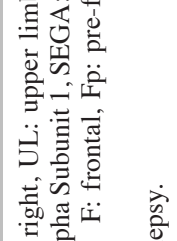 \\
\hline$\stackrel{5}{=}$ & $\stackrel{8}{=}$ & $\stackrel{s}{x}$ & $\stackrel{5}{=}$ & $\stackrel{8}{x}$ & $\stackrel{8}{x}$ & 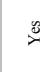 & & & $\frac{8}{5}$ & $\stackrel{8}{\nu}$ & & & & . & $\stackrel{8}{x}$ & & $\stackrel{3}{3}$ & & & & $\stackrel{8}{:}$ & & 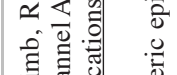 \\
\hline ช & 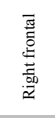 & 总 & 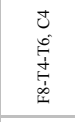 & $\stackrel{n}{n}$ & $\begin{array}{l} \pm \\
\text { है }\end{array}$ & & & & 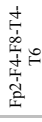 & t & & & 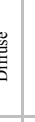 & 善 & 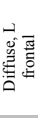 & 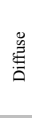 & 善 & 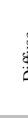 & & & 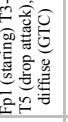 & & 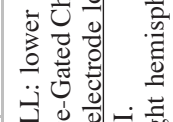 \\
\hline . & . & & & ' & 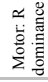 & & & & & & & & & . & & & . & & & & & & \\
\hline ' & . & & & . & ' & & & & & & & & & ' & & & 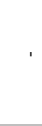 & & & & & & 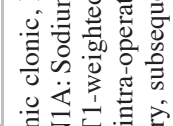 \\
\hline 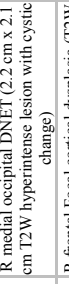 & 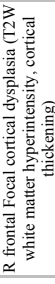 & 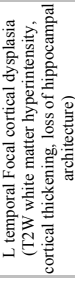 & 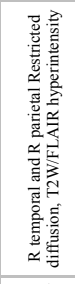 & 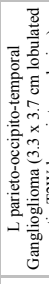 & 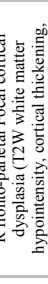 & 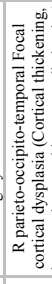 & & & 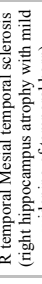 & Ex & & & & 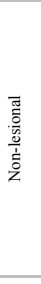 & 跣 & & 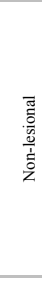 & $\frac{\pi}{2}$ & & & 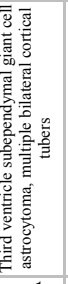 & & 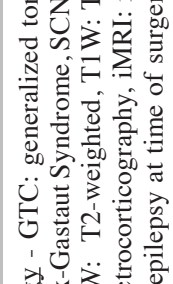 \\
\hline 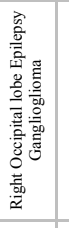 & 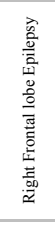 & 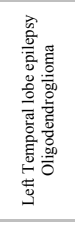 & 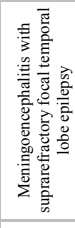 & 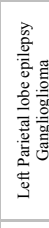 & 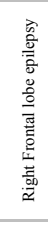 & 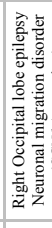 & & & 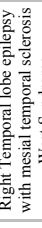 & & . & & 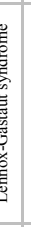 & 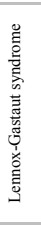 & 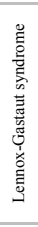 & 竞 & 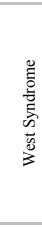 & 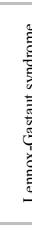 & & & 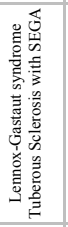 & & 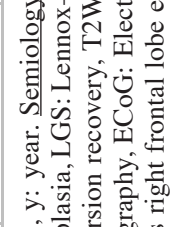 \\
\hline$\underline{\underline{a}}$ & 离 & 产 & 言 & $\Xi$ & 离 & ㅁํㄹ & & & 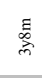 & & & & 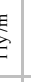 & ह & 赔 & & 蛋 & 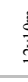 & & & 离 & & \\
\hline$\sqrt[n]{3}$ & $\because$ & $\vec{i}$ & 寒 & $m$ & in & $\because$ & & & $\bar{\omega}$ & 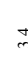 & & & 5 & $\vec{r}$ & $\vec{\infty}$ & 宗 & r & 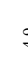 & & & $\stackrel{\infty}{\infty}$ & & \\
\hline 㿣 & $\vec{m}$ & $\stackrel{\mathrm{g}}{\mathrm{g}}$ & 受 & $\Rightarrow$ & ฐ & 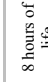 & & & ह & 葴 & & & 密 & 号 & 竧 & ह & 寻 & 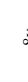 & & & $\Rightarrow$ & & \\
\hline$\Sigma$ & 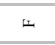 & 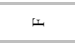 & $\omega$ & $\Sigma$ & $\Sigma$ & $z$ & & & 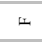 & $t$ & & & $\Sigma$ & $\Sigma$ & $\Sigma$ & 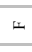 & $\Sigma$ & & & & $\Sigma$ & & \\
\hline$\underline{\simeq}$ & $\underline{0}$ & $=$ & $\stackrel{\infty}{\underline{0}}$ & g & i & $\bar{\sim}$ & & & $\pi$ & 7 & ( & & is & $\therefore$ & $\infty$ & ते & p & 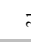 & & & 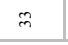 & & \\
\hline
\end{tabular}


Table 2: Overview of epilepsy surgery outcomes at KK Women's and Children's Hospital from 2009-2020, $n=32$

\begin{tabular}{|c|c|c|c|c|c|c|}
\hline $\begin{array}{l}\text { Surgery, } \\
\text { Pathology }\end{array}$ & $\begin{array}{c}\text { Number } \\
\text { performed } \\
\text { at KKH, } \\
\text { n }(\%)\end{array}$ & Indications & $\begin{array}{l}\text { Consistent } \\
\text { predictors of } \\
\text { success }\end{array}$ & $\begin{array}{l}\text { Seizure } \\
\text { Freedom } \\
\text { Rate, } \\
\text { literature }\end{array}$ & $\begin{array}{l}>50 \% \\
\text { seizure } \\
\text { reduction } \\
\text { Rate at } \\
\text { KKH, } \\
\text { n }(\%)\end{array}$ & $\begin{array}{c}\text { Seizure } \\
\text { Freedom } \\
\text { Rate at } \\
\text { KKH, } \\
\text { n }(\%)\end{array}$ \\
\hline $\begin{array}{l}\text { Lesionectomy } \\
\text { Focal Cortical Dysplasia } \\
\text { With Tuberous Sclerosis } \\
\text { With polymicrogyria } \\
\text { DNET } \\
\text { Ganglioglioma }\end{array}$ & $\begin{array}{l}8(\mathbf{2 5 \%}) \\
6 \\
1 \\
1 \\
1 \\
1\end{array}$ & $\begin{array}{l}\text { Well-defined, } \\
\text { radiographically } \\
\text { apparent lesions } \\
\text { (FCD, low } \\
\text { grade tumours, } \\
\text { arteriovenous } \\
\text { malformation) }\end{array}$ & $\begin{array}{l}\text { - Gross total } \\
\text { resection }\end{array}$ & $60-100 \%$ & $8(100 \%)$ & $5(63 \%)$ \\
\hline $\begin{array}{l}\text { Anterior Temporal } \\
\text { Lobectomy } \\
\text { Hippocampal sclerosis } \\
\text { Tuberous Sclerosis } \\
\text { DNET } \\
\text { Ganglioglioma } \\
\text { Oligodendroglioma } \\
\text { Gliosis }\end{array}$ & $\begin{array}{l}1 \\
1 \\
1 \\
1 \\
1 \\
1\end{array}$ & $\begin{array}{l}\text { Small lesions } \\
\text { limited to } \\
\text { temporal lobe } \\
\text { (mesial temporal } \\
\text { sclerosis, FCD, } \\
\text { arteriovenous } \\
\text { malformation, } \\
\text { tuberous sclerosis) }\end{array}$ & $\begin{array}{l}\text { - Visible lesion } \\
\text { - Lack of } \\
\text { secondary } \\
\text { generalization } \\
\text { - Lack of } \\
\text { bilateral } \\
\text { involvement }\end{array}$ & $76 \%$ & $\begin{array}{l}5(83 \%) \\
6(100 \%) \\
\text { after repeat } \\
\quad \text { ATL }\end{array}$ & $\begin{array}{c}5 \\
(83 \%)\end{array}$ \\
\hline $\begin{array}{l}\text { Extratemporal } \\
\text { Lobectomy (Unilobar) } \\
\text { Focal Cortical Dysplasia } \\
\text { Ganglioglioma } \\
\text { Gliosis }\end{array}$ & $\begin{array}{l}4(13 \%) \\
2 \\
1 \\
1\end{array}$ & $\begin{array}{l}\text { Small lesions } \\
\text { away from } \\
\text { temporal lobe } \\
\text { (low-grade } \\
\text { tumours, FCD, }\end{array}$ & $\begin{array}{l}\text { - Short epilepsy } \\
\text { duration } \\
\text { - Lesional } \\
\text { aetiology } \\
\text { - No secondary }\end{array}$ & $56 \%$ & $3(75 \%)^{*}$ & $2(50 \%)$ \\
\hline $\begin{array}{l}\text { Extratemporal } \\
\text { Lobectomy (Multilobar) } \\
\text { Focal Cortical Dysplasia } \\
\text { Sturge Weber Syndrome }\end{array}$ & $3(9 \%)$ & $\begin{array}{l}\text { gliosis, tuberous } \\
\text { sclerosis) }\end{array}$ & $\begin{array}{l}\text { generalization } \\
\text { - Ictal EEG } \\
\text { localization } \\
\text { - Frontal } \\
\text { location }\end{array}$ & & $2(67 \%) \dagger$ & $2(67 \%)$ \\
\hline $\begin{array}{l}\text { Hemispherectomy } \\
\text { Focal Cortical Dysplasia } \\
\text { Hemimegaencephaly }\end{array}$ & $\begin{array}{l}4 \ddagger(13 \%) \\
3 \\
1\end{array}$ & $\begin{array}{l}\text { Large lesions } \\
\text { involving majority } \\
\text { of hemisphere } \\
\text { (large FCD, hemi- } \\
\text { megalencephaly) }\end{array}$ & $\begin{array}{l}\text { - Acquired or } \\
\text { progressive } \\
\text { aetiology } \\
\text { - Unilateral } \\
\text { EEG } \\
\text { involvement } \\
\text { - No history of } \\
\text { other resections }\end{array}$ & $50-85 \%$ & $\begin{array}{l}3(75 \%) \\
4(100 \%) \\
\text { after repeat } \\
\text { hemi- } \\
\text { spherec- } \\
\text { tomy }\end{array}$ & $3(75 \%)$ \\
\hline $\begin{array}{l}\text { Corpus callosotomy } \\
\text { Lennox-Gastaut Syndrome } \\
\text { With SEGA } \\
\text { West Syndrome }\end{array}$ & $\begin{array}{l}9(\mathbf{2 8 \%}) \\
7 \\
1 \\
1\end{array}$ & $\begin{array}{l}\text { Pathologies that } \\
\text { cause significant } \\
\text { drop-attacks, } \\
\text { non-amenable } \\
\text { to potentially- } \\
\text { curative resection }\end{array}$ & $\begin{array}{l}\text { - Infantile } \\
\text { spasms } \\
\text { - Normal MRI } \\
\text { - Short epilepsy } \\
\text { duration } \\
\text { - Complete } \\
\text { callosotomy } \\
\text { - Idiopathic } \\
\text { aetiology }\end{array}$ & $\begin{array}{l}18.8 \% \\
\text { overall, } \\
55.3 \% \\
\text { in drop } \\
\text { attacks }\end{array}$ & $\begin{array}{l}9(100 \%) \\
\text { in drop } \\
\text { attacks }\end{array}$ & $\begin{array}{l}6(67 \%) \\
\text { in drop } \\
\text { attacks }\end{array}$ \\
\hline
\end{tabular}

\footnotetext{
* Patient 7 initially underwent right frontal lobectomy but did not achieve $>50 \%$ reduction in seizures, hence underwent hemispherectomy, and is subsequently seizure free.

$\dagger$ Patient 3 initially underwent right insular, frontal and temporal cortical resection but did not achieve $>50 \%$ reduction in seizures, hence underwent hemispherectomy, and is subsequently seizure free. She was hospitalized for 207 days in total. After the first multilobar resection, she still had persistent seizures, hence underwent a right hemispherectomy 152 days after. After the right hemispherectomy, she was seizure free, and underwent a period of inpatient neuro-rehabilitation. The second longest length of hospital stay was 32 days.

\$ This value includes two patients (Patient 3 and Patient 7) who proceeded with hemispherectomy as second surgeries, as seizures recurred after the first surgeries.
} 

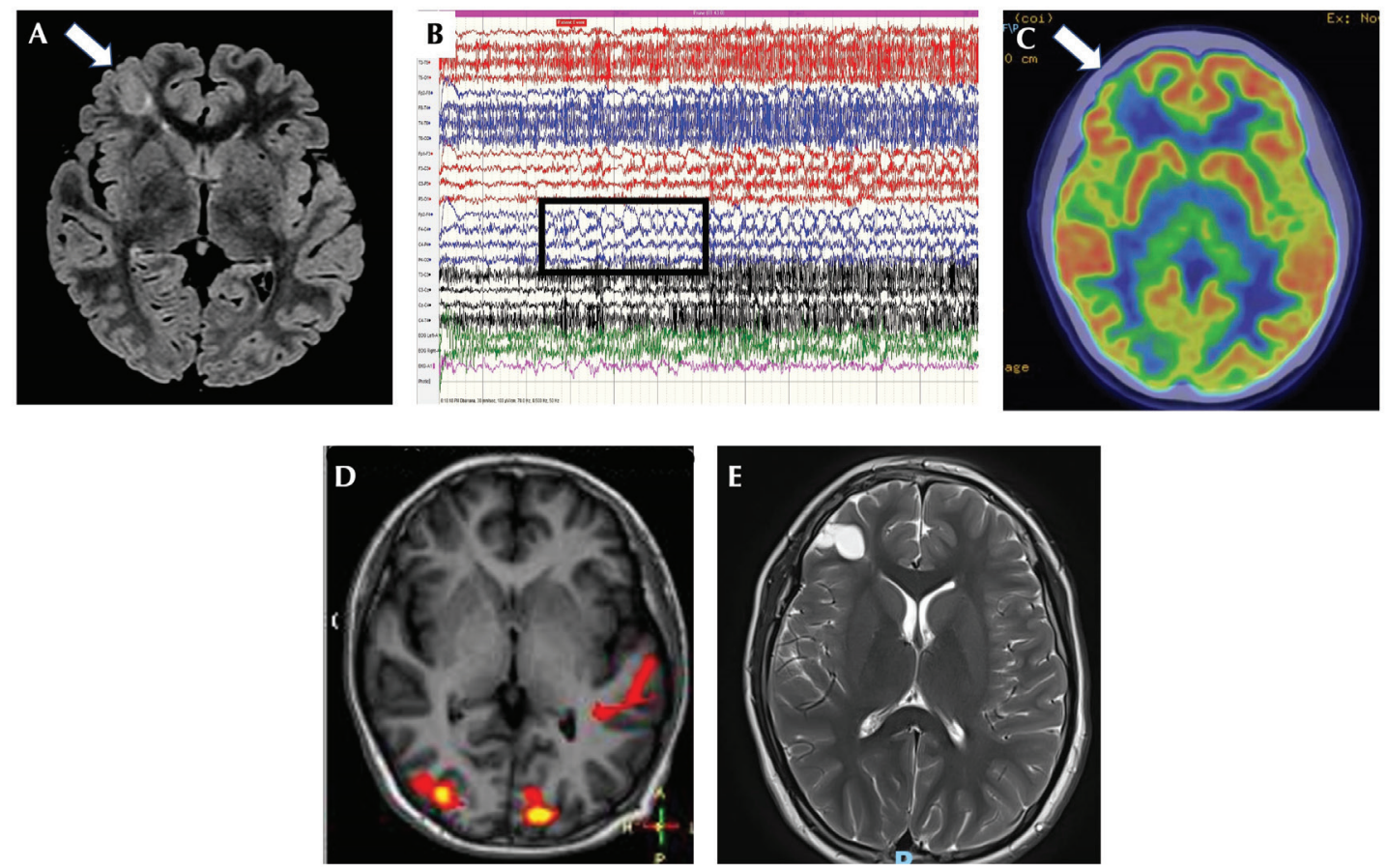

Figure 1. Pre-surgical evaluation for Patient 1.

Representative images from Patient 1, a 12-year-old with Tuberous Sclerosis who underwent lesionectomy for Right Lateral Frontal lobe epilepsy. MRI FLAIR showed a right frontal lesion (A; arrow) that corresponded with F4 discharges on EEG (B; box) and hypometabolism focus on PET (C; arrow). EEG (B) showed rhythmic 2-2.5 Hz delta activity reversing F4 and F3, spreading to involve F8, consistent with a right frontal lobe onset. Clinically, she "raised her upper limbs, turned her head to the right while abducting lower limbs". Functional MRI (colour overlays) performed for pre-surgical planning showed speech localising to the left side (D). Post-surgical T2-weighted MRI showed the excision of right frontal lobe epileptic focus (E).

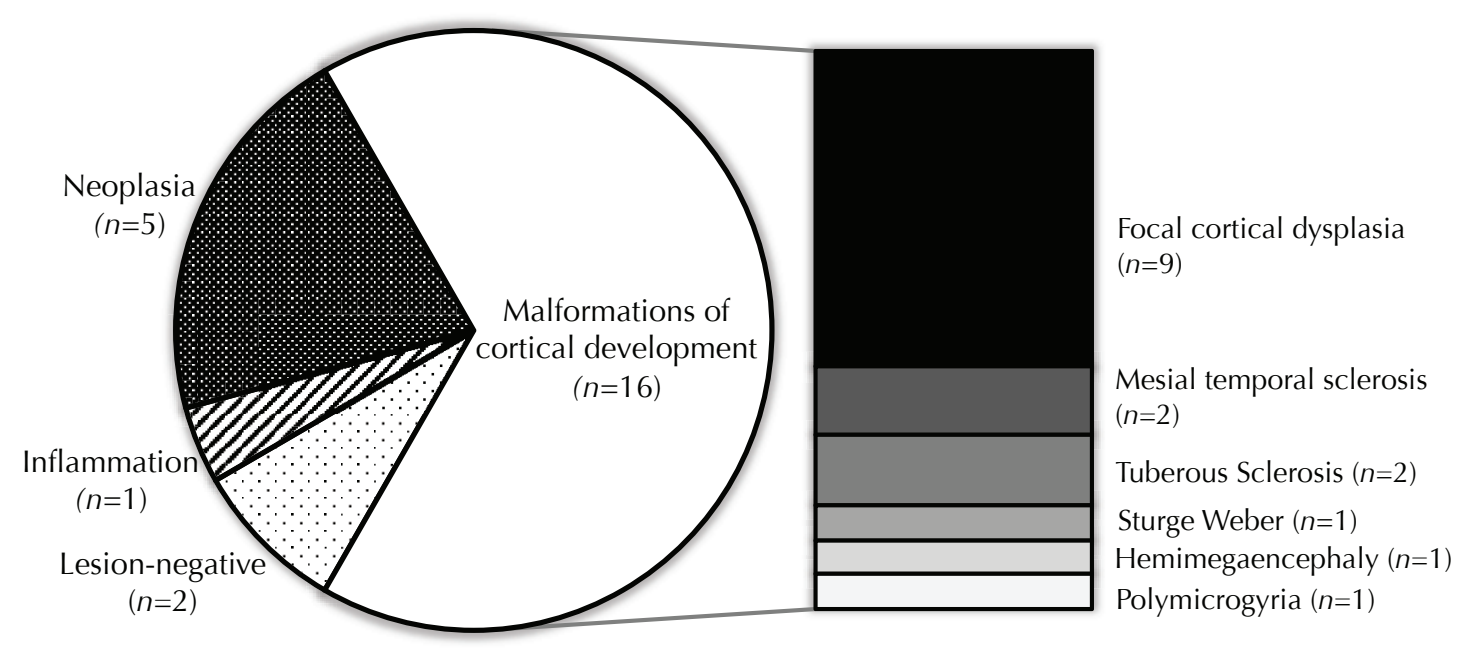

Figure 2. MRI Findings in children with refractory focal epilepsy undergoing resective surgery

Of 24 patients, the most common finding was a malformation of cortical development, followed by benign tumours and one with inflammation (Table 2, Patient 18). Two patients had lesions that were MRI-negative but PET-positive. 
Histopathology in focal epilepsy patients $(n=24)$ revealed 14 FCD (of which two were associated with TS lesions), three ganglioglioma (one WHO Grade I, two WHO Grade II), two DNET, one oligodendroglioma, two gliosis, one showing inflammation, and one SWS malformation.

\section{Post-operative course}

Median hospital stay was ten days (range 4-207 days). Common minor complications were: electrolyte disturbances $(n=11)$, post-operative fever $(n=9)$, nausea and vomiting $(n=6)$, culture-proven infections such as pneumonia or urinary tract infections requiring antibiotics $(n=6)$, Syndrome of Inappropriate Antidiuretic Hormone secretion (SIADH) $(n=3)$, AED-related extravasation $(n=3)$ and rash $(n=3)$. Minor neurological complications were post-operative seizures $(n=9)$ and AED-related drowsiness $(n=5)$.

None of the 15 patients who underwent lesionectomies or anterior temporal lobectomy had motor deficits. One occipital lesionectomy patient had an expected visual scotoma. Nine patients underwent extra-temporal unilobar or multilobar lobectomies and hemispherectomy, with eight having anticipated neurological deficits of hemiparesis, homonymous hemianopia and/or hemispatial neglect.

One patient required debridement for wound dehiscence four months post-operatively, one required evacuation of a tension pneumocephalocele on post-operative day one, one had a right parietal swelling requiring bedside aspiration on post-operative day ten, and surgical evacuation of subgaleal and epidural haematoma on postoperative day 15 . One developed subdural haemorrhage and lacunar infarcts post-corpus callosotomy. There were no post-operative mortalities.

\section{Outcome}

Most patients had improved seizure control (Table 1). Following first surgery, 15/23 (65\%) focal epilepsy patients were seizure-free (Engel Class IA), and 2/23 (9\%) have rare disabling seizures (Engel Class II). Six patients (Patients $3,4,5,6,7,10)$ had seizure recurrence following surgery (Engel Class IIIA), with median time of recurrence of 51 days. Two with hemispherotomies and one with tailored cortical resection to spare motor cortex subsequently underwent anatomical hemispherectomy. Another had extended temporal lobectomy as second surgery. All four of them achieved $>50 \%$ seizure reduction following second surgery, with two becoming seizure-free. Ultimately, 17/23 (74\%) of focal epilepsy patients were seizure-free. Corpus callosotomy reduced drop attacks by $>50 \%$ in $9 / 9(100 \%)$, with $6 / 9$ (67\%) having residual seizures but no further drop attacks and 1 patient completely seizurefree. AEDs were weaned in 21/32 (66\%), with $14 / 32(44 \%)$ on two or fewer AEDs at the last clinic consult.

\section{DISCUSSION}

This audit demonstrates good outcomes in our paediatric epilepsy surgery programmes, comparable to published outcomes by programmes elsewhere. The 23 of 24 patients who underwent resections for focal epilepsy continued follow-up for at least three months. All 23 achieved greater than $50 \%$ seizure reduction, and 17 were seizurefree (Engel Class IA). For patients who underwent corpus callosotomies, 9/9 (100\%) achieved greater than $50 \%$ decrease in drop attacks. Our outcomes were compared to published outcomes in Table $2 .^{20}$

Concerns by patients and caregivers of serious postoperative morbidity and mortality are common and often lead to epilepsy surgery delay. However, mortality in childhood DRE is five to nine times higher than the general population..$^{21,22}$ Seizure freedom is rare in un-operated children with neuroimaging abnormalities, occurring in only $8.6 \%$ of children, ${ }^{23}$ Anecdotal reports from our patients' caregivers also support improved quality of life and learning following successful surgery, suggesting that the risks of continued seizures outweigh risks of surgery. Early epilepsy surgery offers the possibility of reduction or discontinuation of anticonvulsants. AED discontinuation is associated with improved postoperative intelligence ${ }^{24}$, alertness, psychomotor speed, memory and learning. ${ }^{10}$ Most patients continue AEDs for the first six to 24 months after surgery, after which some reduce or withdraw treatment. ${ }^{25}$ Many continue AEDs, albeit with improved seizure control, and in a report of epilepsy surgery for paediatric cerebral malformations, $75 \%$ were still taking anticonvulsants after five years ${ }^{26}$, similar to our institution. Long-term studies do support a higher likelihood of stopping AEDs following epilepsy surgery. A longitudinal study of 60 children from Sweden reported that $86 \%$ of seizure-free children in the surgical group were off AEDs by ten years, compared to none of the non-operated patients. ${ }^{26}$

Temporal lobe epilepsy has a particularly favourable surgical outcome, with resection 
considered the standard of care in drug-refractory cases. Of the six children in our series with sufficient follow-up, $100 \%$ were seizure-free post-surgery. This is comparable with seizure freedom rates of approximately $76 \%$ observed in other studies. ${ }^{27}$

Extra-temporal epilepsy is more prevalent in paediatric patients but is reported to have a lower seizure-freedom rate: $56 \%$ in a systemic review with 1259 paediatric patients. ${ }^{28}$ Among our extra-temporal epilepsy patients, $100 \%$ achieved greater than $50 \%$ seizure reduction, with $10 / 17$ (59\%) achieving Engel Class IA outcome after first surgeries and two more achieving Engel Class IA outcome after second surgery (overall 12/17 (71\%)).

Hemispherectomy is indicated for multilobar cortical dysplasia, hemimegalencephaly, acquired perinatal vascular insults, or progressive lesions such as leptomeningeal angiomas from SWS or Rasmussen's encephalitis. Reported seizurefreedom rates varied from 50-85\% among paediatric patients..$^{20}$ Postoperative hemiparesis and/or homonymous hemianopsia is generally expected. For our hemispherectomy patients, $3 / 4$ (75\%) achieved Engel Class IA seizure-freedom.

DNETs and gangliogliomas are benign tumours that can be highly epileptogenic. DNETs, in particular, tend to be extremely refractory to AEDs. ${ }^{29,30}$ All five of our patients (Patients 8, $14,15,19,22)$ who underwent tumour resection had prior cognitive delay and had failed multiple AEDS. After surgery, all five were seizure-free, with three weaning AEDs. These figures are comparable to results from Ranger and Diosy's 2015 systematic review that demonstrated long-term seizure-freedom in $86 \%$ and seizure improvement in $99 \%$ of the 185 paediatric patients who underwent surgical resection of DNETs. ${ }^{30}$

Corpus callosotomy is beneficial in suitable patients, even if complete seizure freedom rates have been reported to be less than $10-20 \% .^{20,32} \mathrm{We}$ evaluated and selected patients with drop attacks amenable to surgical intervention and 6/9 (67\%) achieved freedom from drop attacks, comparable to $55.3 \%$ demonstrated in a 2018 metanalysis ${ }^{33}$. At our institution, three corpus callosotomies performed before 2010 were partial, due to reported higher risks of transient disconnection syndrome, mutism. ${ }^{9,32}$ From 2010, a decision was made to perform complete corpus callosotomies as we felt these are more likely to make a clinical impact. Studies have reported $88.2 \%$ worthwhile reduction in seizures for patients who had complete callosotomies compared to just
$58.6 \%$ reduction in seizures for those who had partial callosotomies. ${ }^{24,32,34}$ With this decision, we found concomitant improved seizure control. We observed no cases of disconnection syndrome or mutism and we now recommend complete corpus callosotomy to all suitable patients to optimize efficacy and avoid the need for repeat surgery.

Post-operative deficits at our centre were largely as anticipated pre-operatively. These include the one occipital lesionectomy patient with an expected visual scotoma, and the eight out of nine extra-temporal lobectomy and hemispherectomy patients who had anticipated neurological deficits of hemiparesis, homonymous hemianopia and/or hemi-spatial neglect. Post-operative complications at our centre were rare and comparable to what has previously been reported, with $4.5-5.1 \%$ having serious medical or neurological complications. ${ }^{25}$ There were no post-operative mortalities. The four patients (12\%) with unanticipated post-operative complications, such as wound dehiscence, tension pneumocephalocele or surgical related haematomas, received timely management. All four still achieved greater than 50\% reduction in seizure control, and three achieved Engel Class 1A outcome ultimately. In lesionectomies and temporal lobectomies, complications resulting in permanent contralateral weakness or major visual field deficits are reported to be rare, ${ }^{25}$ no unanticipated deficits were seen in our series.

Post-operative seizure recurrence was mainly attributed to initial conservative resection plans. The only independent factor of surgical success is complete resection of both the focal lesion and associated epileptogenic zone. ${ }^{6,28,35,36}$ Other reasons postulated were: development of new epileptogenic zones induced by brain injury during surgery, infection, or microenvironment changes causing alterations in drug-sensitivity in pathological tissues. ${ }^{37}$ To optimize surgical resection and reduce risks of postoperative seizures and repeat surgery, we introduced iMRI into our practice. This was especially useful for lesions that might extend beyond visible margins on MRI, or poorly defined lesions without distinct borders such as FCD or TS. iMRI use has been associated with smaller residual lesions and is protective against poor Engel outcome score. ${ }^{22,25}$ In Sacino et al.'s 2016 study of 29 paediatric patients who underwent resection of FCD or heterotopia localized to eloquent cortex regions, nine of 11 patients (82\%) in the iMRI resection group were seizure free (Engel Class IA) compared with seven of 18 patients (39\%) in control group $(p=0.05) .{ }^{22}$ Eid et al.'s study also 
revealed that iMRI was protective against poor seizure outcome $(p=0.048)$ among 80 paediatric patients who underwent focal epilepsy surgery from 2003 to 2017. Additionally, this study revealed that, while iMRI may prolong mean operative time by 1.2 hours, it was not associated with additional complications. ${ }^{25}$ In our study, seven out of $23(30 \%)$ focal epilepsy patients had iMRI incorporated into their first surgeries. Five of these seven $(71 \%$ ) are now seizure free (Engel Class 1A). An example of iMRI use is presented in Figure 3. Ten out of $16(63 \%)$ focal epilepsy patients who underwent resections without iMRI were seizure free after the first surgery. Some of these surgeries preceded introduction of iMRI, some did not require additional step of iMRI and some were unable to go for iMRI due to logistical reasons. In our experience, tailoring of resection in real time, by the epilepsy neurosurgeon and epileptologist, with iMRI offers value in limiting deficits, improving seizure outcome and reducing risk of re-operation.
One of our patients (Patient 21) harboured a pathogenic SCN1 A mutation. He presented at eight hours of life with focal seizures characterized by eye deviation and tonic stiffening. His seizures proved refractory to several AEDs, and he had up to 30-40 seizures daily. Neuroimaging demonstrated FCD and, following tailored resection, is seizure-free and making developmental progress. Presence of genetic mutations is often taken as a contraindication to epilepsy surgery. These should be weighed up during evaluation, but detailed phenotyping should ultimately drive clinical decision-making.

Our study is limited by small sample size, which does not permit further sub-analysis. Follow-up studies of longer-term seizure control and neuropsychological and social outcomes would also be desirable.

In conclusion, the outcomes of our epilepsy surgery programme are comparable to other centres despite smaller numbers relative to centres overseas. Patients with successful surgical
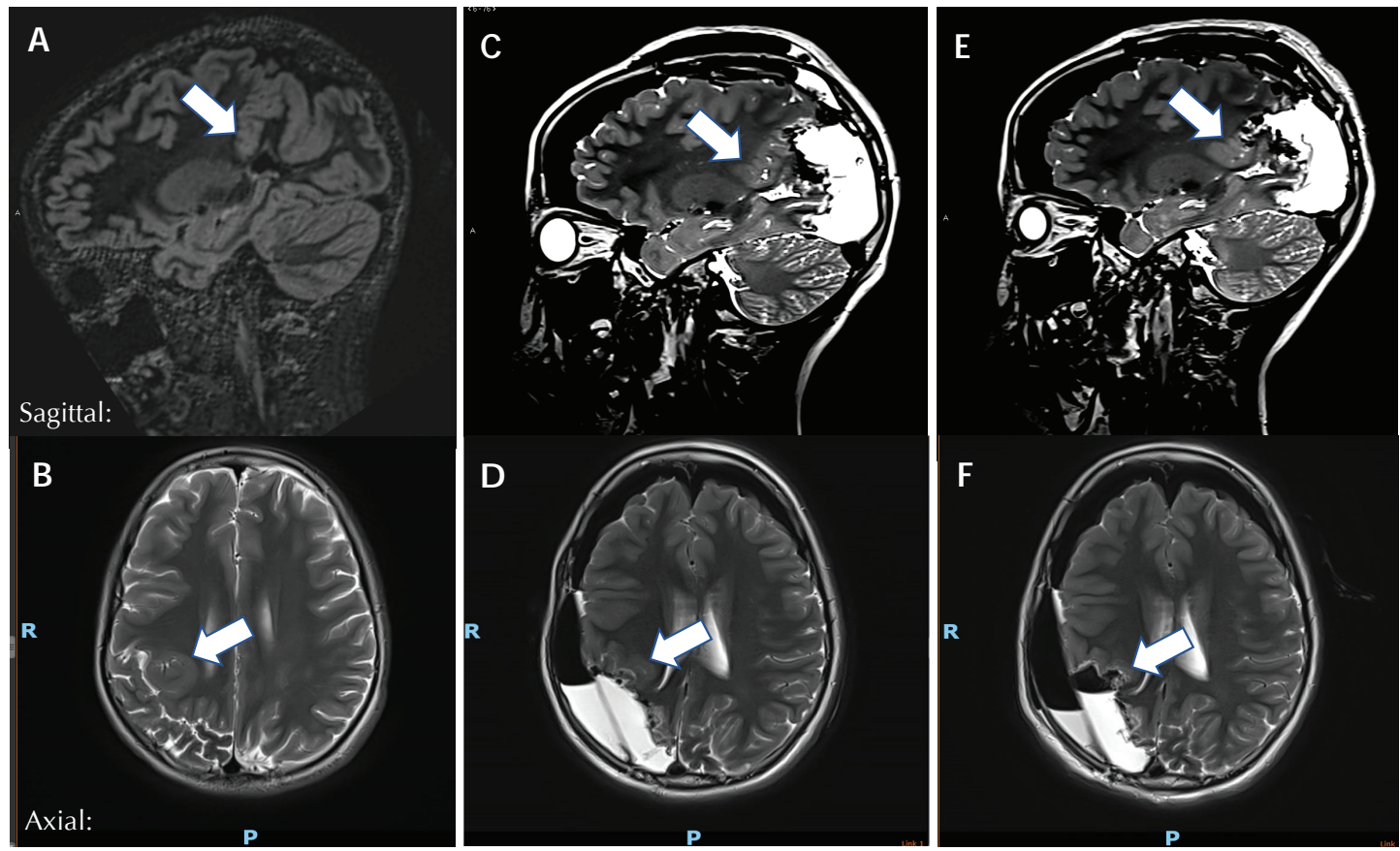

Figure 3. Right parieto-occipital lesionectomy guided by intra-operative MRI.

Patient 24 had drug-resistant focal epilepsy from right parieto-occipital polymicrogyria. Pre-operative MRI showed right parietooccipital lobe polymicrogyria on sagittal image (A; arrow), and corresponding abnormal cortex on axial image (B; arrow). This patient underwent right parieto-occipital lesionectomy at 11 years old. The first intra-operative T2-weighted MRI showed residual abnormal cortex anterior to surgical cavity $(\mathrm{C}$; arrow, D; arrow), further resection of the anterior subcortical margin was performed in the same session (E; arrow, F; arrow). Mild residual abnormal cortex was not shown to be epileptogenic using intra-operative Electrocorticography $(\mathrm{ECoG})$ and decision was made to halt resection at this point so as to preserve motor function. At the six-month follow-up, this patient was seizure free. 
outcome demonstrated better quality of life, concentration, mood, educational and employment opportunities. For well-selected candidates, in whom spontaneous, permanent and complete remission is otherwise rare, epilepsy surgery changes the natural course of disease. iMRI is beneficial in tailoring resection and avoiding reoperation. Patients with positive genetic findings do not have to be excluded from epilepsy surgery if electroclinical phenotype suggests this may be of benefit.

Epilepsy surgery remains an underutilized treatment for children with DRE and should be offered early in suitable patients, rather than as a last resort or not at all. ${ }^{10}$

\section{ACKNOWLEDGEMENT}

We would like to thank KK Women's and Children's Hospital Department of Paediatric Neurology and Prof Lim Shih Hui for their support.

\section{DISCLOSURE}

Financial support: None

\section{Conflict of interest: None}

\section{REFERENCES}

1. Baca CB, Vickrey BG, Caplan R, Vassar SD, Berg AT. Psychiatric and medical comorbidity and quality of life outcomes in childhood-onset epilepsy. Pediatrics 2011;128(6):e1532-1543.

2. Hauser WA. The prevalence and incidence of convulsive disorders in children. Epilepsia 1994;35 (Suppl 2):S1-6.

3. Chan D, Phuah H-K, Ng YL, Choong CT, Lim KW, Goh WHS. Pediatric epilepsy and first afebrile seizure in Singapore: Epidemiology and investigation yield at presentation. J Child Neurol 2010;25(10):1216-22.

4. Kwan P, Arzimanoglou A, Berg AT, et al. Definition of drug resistant epilepsy: consensus proposal by the ad hoc Task Force of the ILAE Commission on Therapeutic Strategies. Epilepsia 2010;51(6):1069-77.

5. Berg AT, Vickrey BG, Testa FM, et al. How long does it take for epilepsy to become intractable? A prospective investigation. Ann Neurol 2006;60(1):739.

6. Engel J, Pedley TA. Epilepsy: A comprehensive textbook, 2nd Edition. Lippincott Williams \& Wilkins; 2008

7. Braun KPJ, Cross JH. Pediatric epilepsy surgery: the earlier the better. Expert Rev Neurother 2018;18(4):261-3.

8. Cross JH, Jayakar P, Nordli D, et al. Proposed criteria for referral and evaluation of children for epilepsy surgery: recommendations of the Subcommission for Pediatric Epilepsy Surgery. Epilepsia 2006;47(6):9529.
9. Sugano H, Arai H. Epilepsy surgery for pediatric epilepsy: Optimal timing of surgical intervention. Neurol Med Chir (Tokyo) 2015;55(5):399-406.

10. Lee KH, Lee YJ, Seo JH, Baumgartner JE, Westerveld M. Epilepsy surgery in children versus adults. $J$ Korean Neurosurg Soc 2019;62(3):328-35.

11. Wiebe S, Blume WT, Girvin JP, Eliasziw M, Effectiveness and Efficiency of Surgery for Temporal Lobe Epilepsy Study Group.A randomized, controlled trial of surgery for temporal-lobe epilepsy. $N$ Engl $J$ Med 2001;345(5):311-8.

12. Dwivedi R, Ramanujam B, Chandra PS, et al. Surgery for drug-resistant epilepsy in Children. $N$ Engl J Med 2017;377(17):1639-47.

13. Asadi-Pooya AA, Malekmohamadi Z, Kamgarpour A, et al. Corpus callosotomy is a valuable therapeutic option for patients with Lennox-Gastaut syndrome and medically refractory seizures. Epilepsy Behav 2013;29(2):285-8.

14. Thuy Le MA,Fong SL, Lim KS, et al. Underutilization of epilepsy surgery in ASEAN countries. Seizure 2019;69:51-6.

15. Scheffer IE, Berkovic S, Capovilla G, et al. ILAE classification of the epilepsies: Position paper of the ILAE Commission for Classification and Terminology. Epilepsia 2017;58(4):512-21.

16. Grinnon ST, Miller K, Marler JR, et al. National Institute of Neurological Disorders and Stroke Common Data Element Project - approach and methods. Clin Trials Lond Engl 2012;9(3):322-9.

17. Blümcke I, Thom $\mathrm{M}$, Aronica $\mathrm{E}$, et al. The clinicopathologic spectrum of focal cortical dysplasias: a consensus classification proposed by an ad hoc Task Force of the ILAE Diagnostic Methods Commission. Epilepsia 2011;52(1):158-74.

18. Louis DN, Perry A, Reifenberger G, et al. The 2016 World Health Organization Classification of Tumors of the Central Nervous System: a summary. Acta Neuropathol (Berl) 2016;131(6):803-20.

19. Engel J, ed: Surgical treatment of the epilepsies. 2nd ed. New York: Raven Press; 1993. 786.

20. Dallas J, Englot DJ, Naftel RP. Neurosurgical approaches to pediatric epilepsy: Indications, techniques, and outcomes of common surgical procedures. Seizure 2018; 77:76-85.

21. Hader WJ, Tellez-Zenteno J, Metcalfe A, et al. Complications of epilepsy surgery-A systematic review of focal surgical resections and invasive EEG monitoring. Epilepsia 2013;54(5):840-7.

22. Sacino MF, Ho CY, Murnick J, Keating RF, Gaillard $\mathrm{WD}$, Oluigbo CO. The role of intraoperative MRI in resective epilepsy surgery for peri-eloquent cortex cortical dysplasias and heterotopias in pediatric patients. Neurosurg Focus 2016;40(3):E16.

23. Sperling MR. The consequences of uncontrolled epilepsy. CNS Spectr 2004;9(2):98-101, 106-9.

24. Boshuisen $\mathrm{K}$, van Schooneveld MMJ, Uiterwaal CSPM, et al. Intelligence quotient improves after antiepileptic drug withdrawal following pediatric epilepsy surgery: IQ after AED withdrawal. Ann Neurol 2015;78(1):104-14.

25. Eid H, Crevier-Sorbo G, Moreau JT, et al. Eight-year experience with 3-T intraoperative MRI integration 
in focal pediatric epilepsy surgery: Impact on extent of resection, residual volumes, and seizure outcomes. Am J Roentgenol 2020;214(6):1343-51.

26. Edelvik A, Rydenhag B, Olsson I, et al. Long-term outcomes of epilepsy surgery in Sweden: a national prospective and longitudinal study. Neurology 2013;81(14):1244-51.

27. Englot DJ. A modern epilepsy surgery treatment algorithm: Incorporating traditional and emerging technologies. Epilepsy Behav 2018;80:68-74.

28. Englot DJ, Breshears JD, Sun PP, Chang EF, Auguste KI. Seizure outcomes after resective surgery for extra-temporal lobe epilepsy in pediatric patients. $J$ Neurosurg Pediatr 2013;12(2):126-33.

29. Bilginer B, Yalnizoglu D, Soylemezoglu F, et al. Surgery for epilepsy in children with dysembryoplastic neuroepithelial tumor: clinical spectrum, seizure outcome, neuroradiology, and pathology. Childs Nerv Syst 2009;25(4):485-91.

30. Ranger A, Diosy D. Seizures in children with dysembryoplastic neuroepithelial tumors of the brain-A review of surgical outcomes across several studies. Childs Nerv Syst 2015;31(6):847-55.

31. Jalilian L, Limbrick DD, Steger-May K, Johnston J, Powers AK, Smyth MD. Complete versus anterior two-thirds corpus callosotomy in children: analysis of outcome. J Neurosurg Pediatr 2010;6(3):257-66.

32. Chan AY, Rolston JD, Lee B, Vadera S, Englot DJ. Rates and predictors of seizure outcome after corpus callosotomy for drug-resistant epilepsy: a meta-analysis. J Neurosurg 2018;1-10.

33. Shim KW, Lee YM, Kim HD, Lee JS, Choi JU, Kim DS. Changing the paradigm of 1-stage total callosotomy for the treatment of pediatric generalized epilepsy. J Neurosurg Pediatr 2008;2(1):29-36.

34. Paolicchi JM, Jayakar P, Dean P, et al. Predictors of outcome in pediatric epilepsy surgery. Neurology 2000;54(3):642-7.

35. Krsek P, Maton B, Jayakar $\mathrm{P}$, et al. Incomplete resection of focal cortical dysplasia is the main predictor of poor postsurgical outcome. Neurology 2009;72(3):217-23

36. Laxer KD, Trinka E, Hirsch LJ, et al. The consequences of refractory epilepsy and its treatment. Epilepsy Behav 2014;37:59-70. 